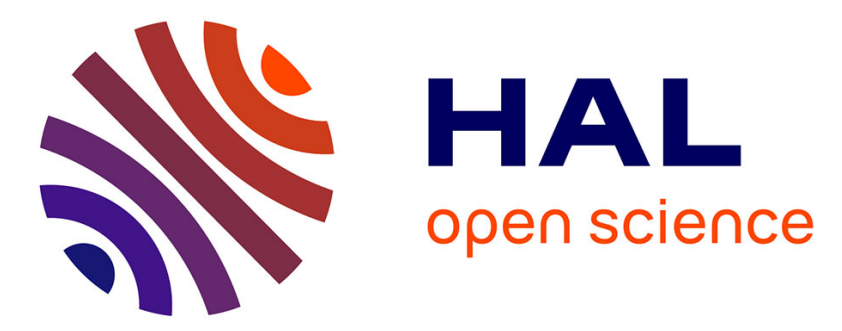

\title{
Stabilité mécanique d'une phase liquide comprise dans un revêtement composite de protection du niobium contre l'oxydation - I. Cas d'une couche de verre visqueux déposée sur un substrat solide
}

J. Gillot, P. Stecher, B. Lux

\section{To cite this version:}

J. Gillot, P. Stecher, B. Lux. Stabilité mécanique d'une phase liquide comprise dans un revêtement composite de protection du niobium contre l'oxydation - I. Cas d'une couche de verre visqueux déposée sur un substrat solide. Revue de Physique Appliquée, 1970, 5 (3), pp.559-564. 10.1051/rphysap:0197000503055900 . jpa-00243428

\section{HAL Id: jpa-00243428 https://hal.science/jpa-00243428}

Submitted on 1 Jan 1970

HAL is a multi-disciplinary open access archive for the deposit and dissemination of scientific research documents, whether they are published or not. The documents may come from teaching and research institutions in France or abroad, or from public or private research centers.
L'archive ouverte pluridisciplinaire HAL, est destinée au dépôt et à la diffusion de documents scientifiques de niveau recherche, publiés ou non, émanant des établissements d'enseignement et de recherche français ou étrangers, des laboratoires publics ou privés. 


\title{
STABILITÉ MÉCANIQUE D'UNE PHASE LIQUIDE COMPRISE DANS UN REVÊTEMENT COMPOSITE DE PROTECTION DU NIOBIUM CONTRE L'OXYDATION
}

\section{CAS D'UNE COUCHE DE VERRE VISQUEUX DÉPOSÉE SUR UN SUBSTRAT SOLIDE}

\author{
par J. GILLOT, P. STECHER, B. LUX \\ Institut Battelle, Genève, Suisse
}

\begin{abstract}
Two processes of viscous flow occur in a glass layer of constant thickness deposited on a solid substrate that is perfectly wetted by the glass : viz. flow of the glass caused by accelerations parallel to the layer and self healing of the cracks by capillary forces. The study of these flows and the comparison of their time constants indicate in which conditions the layer can satisfy both requirements of mechanical stability and fast self healing of the cracks. Simultaneous satisfaction of both requirements is not possible in the case of a glass layer deposited on a gas turbine blade, but is possible in the case of a layer deposited on a static part that operates at constant temperature.
\end{abstract}

1. Introduction. - Les revêtements de protection des métaux contre l'oxydation sont, en général, des couches solides compactes. Cependant, l'emploi de telles couches ne permet pas toujours d'obtenir les caractéristiques voulues. L'adjonction d'une phase liquide peut parfois résoudre ce problème, grâce à la mobilité ainsi conférée à certains constituants du revêtement. Ces constituants peuvent alors assumer des fonctions telles que le remplissage et la guérison des fissures. Néanmoins, leur mobilité fait apparaître un nouveau problème : celui de leur rétention dans les diverses conditions d'utilisation de la pièce recouverte.

C'est ce problème de la stabilité mécanique d'une phase liquide comprise dans un revêtement composite qui va être étudié dans la première partie de ce mémoire, dans le cas particulier d'un revêtement de protection $\mathrm{du}$ niobium contre l'oxydation qui comprend une couche d'un verre visqueux déposée à la surface d'un solide compact. Le cas d'un revêtement constitué d'une couche de solide poreux imprégnée d'un alliage métallique liquide à faible viscosité fera l'objet de la deuxième partie de ce mémoire. La réalisation, les caractéristiques physico-chimiques et les performances de ces revêtements ont été décrites par ailleurs $[1,2,3]$.

Les sollicitations auxquelles sont soumis les revêtements dans divers cas d'applications possibles du niobium ou de ses alliages sont indiquées dans le tableau 1. Seules seront ici considérées les sollicitations qui sont des accélérations qui peuvent être dues à la pesanteur, à une force centrifuge ou à des vibrations.
Le problème est de déterminer dans quelles conditions le verre est assez visqueux pour ne pas s'écouler sensiblement sous l'effet de l'accélération, mais cependant assez fluide pour que les fissures éventuelles du verre puissent se reboucher sous l'effet de la force de capillarité.

On va donc calculer d'une part la vitesse d'écoulement sous l'effet de l'accélération, d'autre part la vitesse de guérison d'une fissure. La comparaison de ces deux vitesses permettra de prévoir dans quelles conditions la couche de verre pourra à la fois rester stable et assurer la guérison de ses fissures éventuelles.

2. Calcul de l'écoulement de la couche sous l'effet d'une accélération. - Le problème est défini par les hypothèses suivantes :

- l'accélération est parallèle à la couche de verre, - le substrat est plan, de dimensions infinies dans les directions $x$ et $y$,

- la couche a une épaisseur constante $e$, tonien,

- le verre a un comportement rhéologique new-

- on étudie l'écoulement en régime permanent.

Pour calculer la vitesse d'écoulement, on considère un élément de volume $\mathrm{d} x, \mathrm{~d} y, \mathrm{~d} z$, situé au point $(x, y, z)$ (Fig. 1).

La force sur cet élément engendrée par l'accélération est :

$$
\mathrm{d} F=\gamma \rho \mathrm{d} x \mathrm{~d} y \mathrm{~d} z
$$

où $\gamma$ est l'accélération et $\rho$ la masse spécifique du verre. 
TABleau I

Applications possibles du niobium ou de ses alliages et sollicitations exercées sur le revêtement

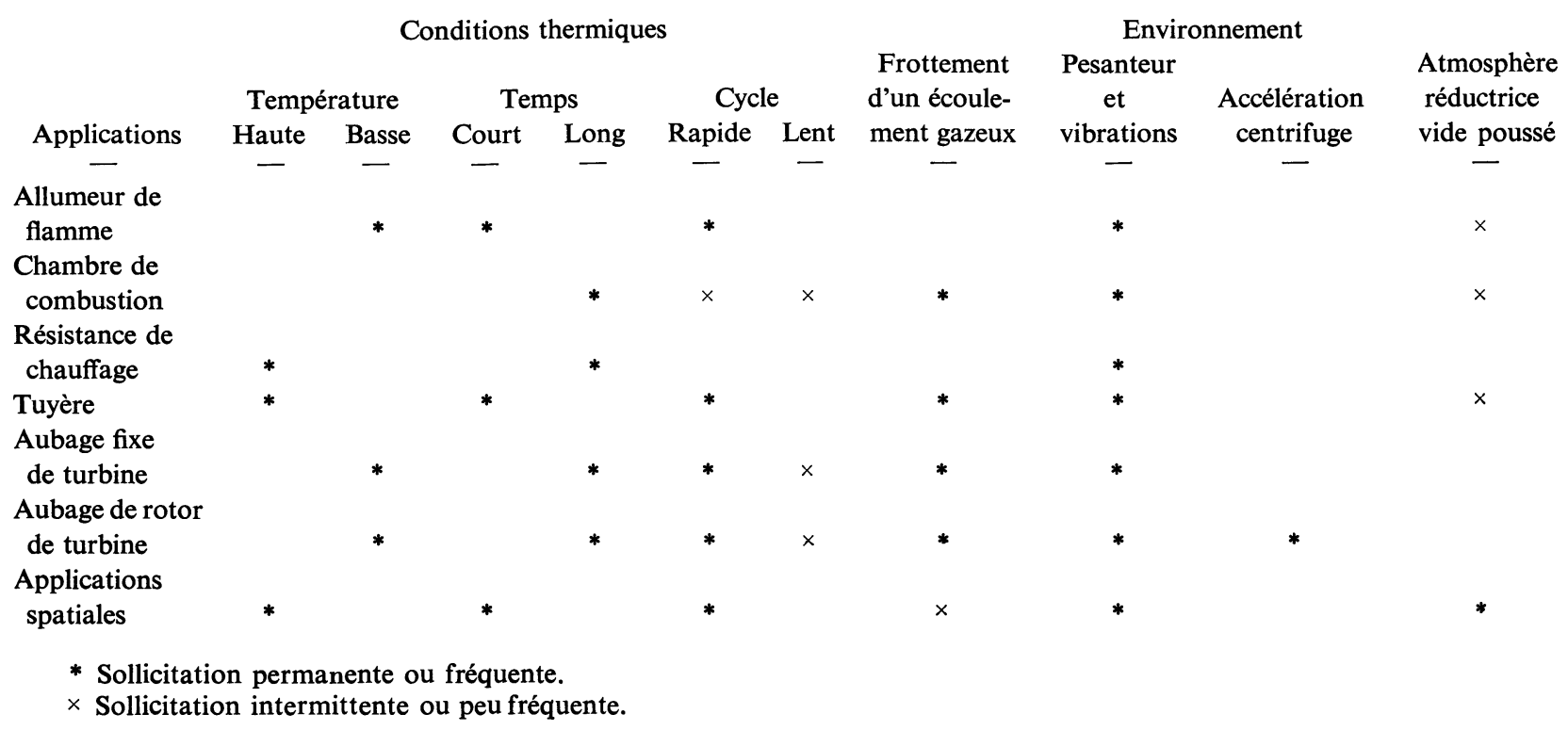

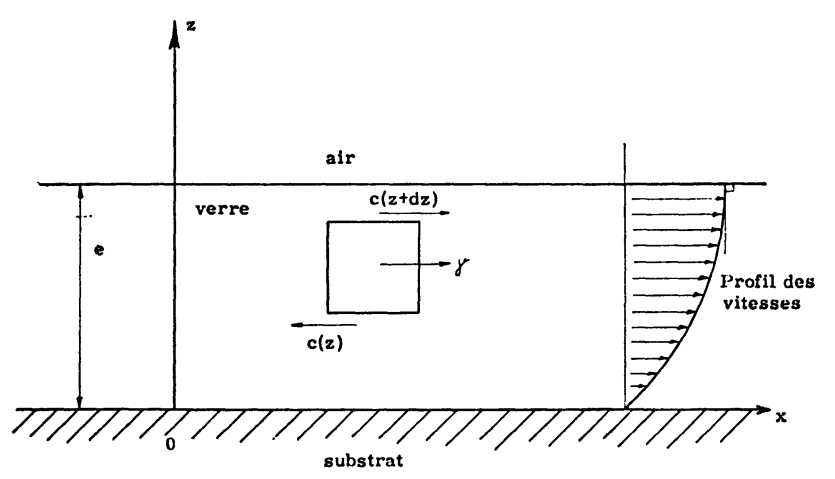

Fig. 1. - Ecoulement de la couche sous l'effet de la force centrifuge.

Elle est équilibrée par les contraintes de cisaillement $c$ sur les faces de cotes $z+\mathrm{d} z$ et $z$ :

$$
\mathrm{d} x \mathrm{~d} y[c(z+\mathrm{d} z)-c(z)]+\mathrm{d} F=0
$$

d'où

$$
\frac{\mathrm{d} c}{\mathrm{~d} z}=-\gamma \rho
$$

et

$$
c=-\gamma \rho\left(z-z_{0}\right) .
$$

A la surface libre $z=e$ et $c=0$

$$
\text { d'où } \quad c=\gamma \rho(e-z) \text {. }
$$

D'après la relation de définition de la viscosité $\mu$ :

$$
c=\mu \frac{\mathrm{d} v}{\mathrm{~d} z} \quad \frac{\mathrm{d} v}{\mathrm{~d} z}=\frac{\gamma \rho}{\mu}(e-z)
$$

où $v$ est la vitesse de déplacement.
En intégrant :

$$
v=\frac{\gamma \rho}{\mu}\left(e z-\frac{z^{2}}{2}+\mathrm{k}\right)
$$

où $\mathrm{k}$ est une constante d'intégration.

La vitesse est nulle au contact avec le substrat. Donc pour $z=0, v=0$, d'où

$$
v=\frac{\gamma \rho}{\mu}\left(e z-\frac{z^{2}}{2}\right) \text {. }
$$

La vitesse maximum est la vitesse à la surface de la couche :

$$
v_{\max }=\frac{\gamma \rho e^{2}}{2 \mu} .
$$

La vitesse moyenne de la couche est :

$$
v_{\text {moy }}=\frac{1}{e} \int_{0}^{e} v(z) \mathrm{d} z=\frac{\gamma \rho e^{2}}{3 \mu} .
$$

On peut considérer que le déplacement risque d'être dangereux dès qu'il est égal à l'épaisseur de la couche :

(La couche risque de s'amincir dangereusement sur ses bords, ou sur les rugosités ou arêtes vives de la couche de base.)

Ceci est atteint au bout d'un temps $t_{e}$ :

$$
t_{e}=\frac{e}{v_{\mathrm{moy}}}=\frac{3 \mu}{\gamma \rho e} \text {. }
$$

3. Evaluation de la durée nécessaire à la guérison d'une fissure sous l'effet de la tension superficielle. 3.1 DÉFINITION DU PROBLÈME. - On considère ici une couche uniforme d'un liquide visqueux newtonien placée sur un substrat rigide plan de dimensions 
infinies que le liquide mouille parfaitement. Cette couche est interrompue sur toute son épaisseur par une fissure rectiligne à profil initialement rectangulaire et de longueur infinie (Fig. 2). On suppose que la largeur $f$ de la fissure n'est pas supérieure à l'épaisseur $e$ de la couche; on suppose également que les forces dues aux accélérations extérieures sont négligeables et que le mouvement est assez lent pour que les forces d'inertie soient négligeables.

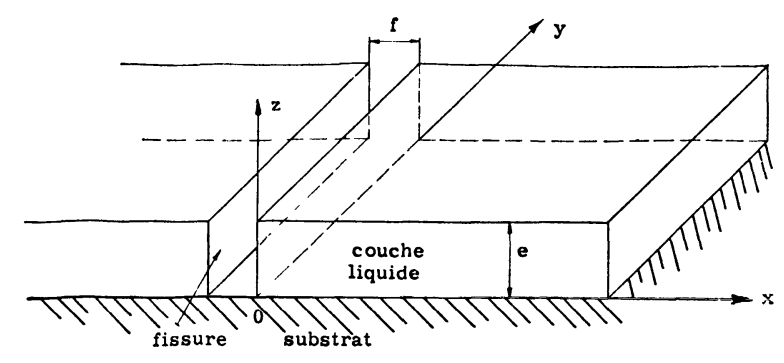

Fig. 2. - Vue générale de la fissure.

La fissure va se refermer sous l'effet de la tension superficielle du liquide. Les différentes étapes de cette guérison sont représentées sur la figure 3 . Le système va évoluer spontanément dans le sens d'une diminution de son énergie, c'est-à-dire d'une diminution de la surface libre du verre. On peut imaginer intuitivement qu'aux temps successifs $t_{1}, t_{2}, t_{3}$ et $t_{4}$, on aura les profils représentés par les courbes 1 , $2,3,4$.

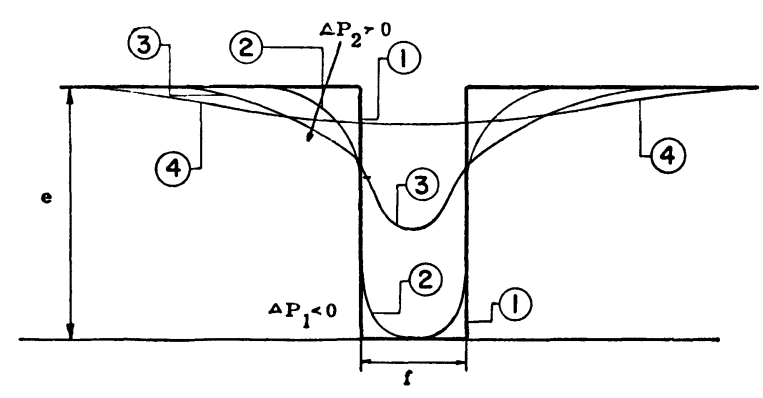

FIg. 3. - Coupe de la fissure aux temps $t_{1}, t_{2}, t_{3}, t_{4}$.

La tension superficielle du liquide provoque l'apparition dans le liquide, au fond de la fissure, d'une diminution de pression par rapport à l'atmosphère ambiante, c'est-à-dire encore par rapport à la pression régnant dans la couche à grande distance de la fissure.

Elle provoque également l'apparition d'un excès de pression aux lèvres de la fissure. C'est sous l'effet de ces différences de pression que la fissure va se refermer. La guérison de la fissure va libérer une énergie proportionnelle à la diminution de la surface du liquide. On peut estimer qu'à mesure que la fissure se remplira, les rayons de courbure augmenteront et qu'ainsi la surface libre du liquide diminuera de plus en plus lentement.
On va évaluer, par un calcul approché, la vitesse de remplissage de la fissure dans la première phase du remplissage, quand la forme de la fissure n'a pas encore été sensiblement modifiée. La figure 4 représente cette étape du remplissage. On en déduira l'ordre de grandeur du temps mis par la fissure pour se refermer.

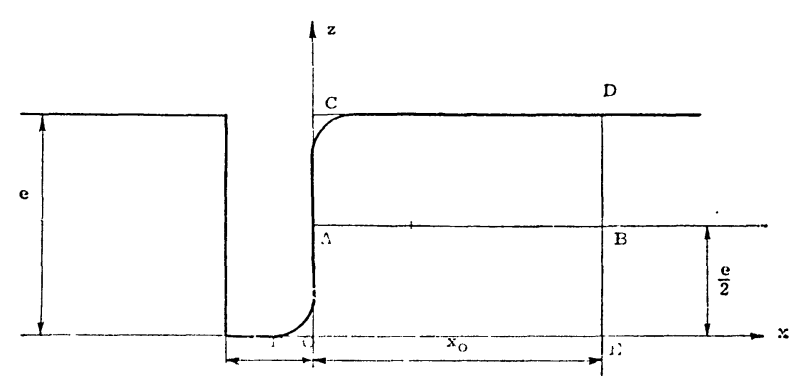

FIG. 4. - Coupe schématique de la fissure.

3.2 Equations DE BASE. - Considérons (Fig. 4) la couche d'un côté de la fissure et le plan de cote $z=e / 2$ passant par $\mathrm{A}$, ainsi qu'un plan parallèle à $y \mathrm{Oz}$ qui coupe la couche en $\mathrm{DBE}$ avec $\mathrm{AB}=x_{0}$.

On écrit les équations d'équilibre pour la section ABDC en considérant que les forces d'inerties sont négligeables car les vitesses atteintes sont trop faibles.

On utilise pour cela les notations suivantes :

$-\sigma$ : tension superficielle du liquide;

$-C_{\mathrm{AB}}$ : contrainte de cisaillement parallèle à $\mathrm{AB}$ sur le plan $\mathrm{AB} y$;

$-P_{\mathrm{AB}}$ : contrainte perpendiculaire au plan $\mathrm{AB} y$. $P_{\mathrm{AB}}$ est positif s'il s'agit d'une compression.

Forces parallèles à $O x$ :

$$
\sigma+\int_{\mathrm{A}}^{\mathrm{B}} C_{\mathrm{AB}} \mathrm{d} x-\int_{\mathbf{B}}^{\mathrm{D}} P_{\mathrm{BD}} \mathrm{d} z=0
$$

Forces parallèles à $O z$ :

$$
-\sigma+\int_{\mathbf{A}}^{\mathbf{B}} P_{\mathrm{AB}} \mathrm{d} x+\int_{\mathbf{B}}^{\mathrm{D}} C_{\mathrm{BD}} \mathrm{d} z=0 .
$$

Moment en A :

$$
\begin{aligned}
& -\sigma \frac{e}{2}+\int_{\mathrm{A}}^{\mathrm{B}} x P_{\mathrm{AB}} \mathrm{d} x+ \\
& \quad+\int_{\mathrm{B}}^{\mathrm{D}}\left(z-\frac{e}{2}\right) P_{\mathrm{BD}} \mathrm{d} z+x_{0} \int_{\mathrm{B}}^{\mathrm{D}} C_{\mathrm{BD}} \mathrm{d} z=0 .
\end{aligned}
$$

On suppose maintenant que le plan $\mathrm{BD}$ est très éloigné de la fissure, c'est-à-dire $x_{0} / e$ très grand.

A grande distance, la couche n'est pas perturbée par la fissure. Les actions de la surpression à la lèvre de la fissure et la dépression à sa base se compensent à grande distance et on a $P_{\mathrm{BD}}=0$.

Ceci est confirmé par le fait expérimental que, quand un liquide mouille un substrat, il s'y étale jusqu'à avoir une épaisseur théoriquement nulle : il n'y a pas de surpression qui compense la force de pesanteur 
comme ce serait le cas avec un liquide qui ne mouille pas le substrat.

De même, on suppose que les cisaillements sont nuls à grande distance de la fissure : $C_{\mathrm{BD}}=0$ et que leur moment est nul : $x_{0} C_{\mathrm{BD}}=0$.

D'ailleurs l'hypothèse que les vitesses sont nulles à grande distance de la fissure entraîne que leurs dérivées sont nulles et que les cisaillements qui sont proportionnels à ces dérivées sont nuls.

3.3 Calcul de la vitesse D'ÉCoulement eN A. (1) devient :

$$
-\sigma=\int_{\mathrm{A}}^{\mathrm{B}} C_{\mathrm{AB}} \mathrm{d} x .
$$

Or, si $v_{x}$ et $v_{z}$ sont les composantes de la vitesse parallèles à $O x$ et à $O z$ et $\mu$ la viscosité du verre,

$$
C_{\mathrm{AB}}=-\mu\left(\frac{\partial v_{x}}{\partial z}+\frac{\partial v_{z}}{\partial x}\right) .
$$

Si on suppose que la largeur de la fissure est au plus du même ordre de grandeur que l'épaisseur de la couche, les déplacements dans la direction $O x$ seront au plus du même ordre de grandeur que ceux dans la direction $O z$. Au début du remplissage de la fissure, quand sa paroi est encore parallèle à $O z$, on peut supposer que le déplacement se fait principalement dans la direction $\mathrm{Oz}$.

On a alors :

$$
C_{\mathrm{AB}}=-\mu \frac{\mathrm{d} v_{z}}{\mathrm{~d} x}
$$

L'équation (4) devient :

$$
\begin{aligned}
-\sigma & =\int_{\mathrm{A}}^{\mathrm{B}}(-\mu) \frac{\mathrm{d} v_{z}}{\mathrm{~d} x} \mathrm{~d} x \\
\frac{\sigma}{\mu} & =v_{z}(\mathrm{~B})-v_{z}(\mathrm{~A}) .
\end{aligned}
$$

A l'infini, la couche est au repos : $v_{z}(\mathrm{~B})=0$

$$
v_{z}(\mathrm{~A})=v_{\mathrm{A}}=-\frac{\sigma}{\mu}
$$

3.4 Evaluation DU FLUX DE volume. - Il faut maintenant évaluer sur quelle distance dans la direction $O x$ ce mouvement se produit.

L'hypothèse que la couche est au repos à l'infini permet de simplifier (2) et (3) :

$$
\begin{aligned}
& \int_{\mathrm{A}}^{\mathrm{B}} P_{\mathrm{AB}} \mathrm{d} x=\sigma \\
& \int_{\mathrm{A}}^{\mathrm{B}} x P_{\mathrm{AB}} \mathrm{d} x=\sigma \frac{e}{2} .
\end{aligned}
$$

L'abscisse du centre de gravité de la distribution de pression $P_{\mathrm{AB}}$ est donc

$$
\frac{\int_{\mathrm{A}}^{\mathrm{B}} x P_{\mathrm{AB}} \mathrm{d} x}{\int_{\mathrm{A}}^{\mathrm{B}} P_{\mathrm{AB}} \mathrm{d} x}=\frac{e}{2} .
$$

L'ordre de grandeur de la distance dans la direction $O x$ sur laquelle le mouvement se produit est donc $e$.

Supposons que la distribution de vitesse $v_{z}$ sur AB suive la loi

$$
v=v_{\mathrm{A}} \exp \left(-2 \frac{x}{e}\right)
$$

L'abscisse du centre de gravité de cette distribution sera également :

$$
\frac{\int_{0}^{\infty} x v \mathrm{~d} x}{\int_{0}^{\infty} v \mathrm{~d} x}=\frac{e}{2} .
$$

Le mouvement provoquera un flux de volume à travers le plan $\mathrm{AB} y$ et sur une profondeur $\mathrm{d} y$ :

$$
\begin{aligned}
\frac{\mathrm{d} V}{\mathrm{~d} t}=\mathrm{d} y \int_{\mathrm{A}}^{\mathrm{B}} v \mathrm{~d} x=v_{\mathrm{A}} \mathrm{d} y \int_{0}^{\infty} & \exp \left(-2 \frac{x}{e}\right) \mathrm{d} x= \\
= & \frac{e}{2} v_{\mathrm{A}} \mathrm{d} y=-\frac{e}{2} \frac{\sigma}{\mu} \mathrm{d} y .
\end{aligned}
$$

En considérant seulement les valeurs absolues :

$$
\frac{\mathrm{d} V}{\mathrm{~d} t}=\frac{e}{2} \frac{\sigma}{\mu} \mathrm{d} y
$$

3.5 Evaluation DE LA CONSTANTE DE TEMPS DU REMPlisSAge DE LA FISSURE. - Pour remplir la fissure sur une hauteur $\mathrm{d} z$, il faut un apport de volume $(f / 2) \mathrm{d} z \mathrm{~d} y$, ce qui nécessite un temps

$$
\mathrm{d} t=\frac{f}{2} \mathrm{~d} z \mathrm{~d} y \frac{\mathrm{d} t}{\mathrm{~d} V}=\frac{f}{e} \frac{\mu}{\sigma} \mathrm{d} z .
$$

Ceci donne l'allure de guérison de la fissure au début de cette guérison.

A mesure que la fissure se remplit et que les courbures de la surface diminuent, l'allure de remplissage diminue. L'allure de remplissage initiale permet de calculer une sorte de constante de temps $t_{f}$ pour la guérison, c'est-à-dire le remplissage sur la profondeur totale $e$ :

$$
t_{f}=e \frac{\mathrm{d} t}{\mathrm{~d} z}=\frac{f \mu}{\sigma}=t_{f}
$$

Le calcul précédent n'a pu être mené à bien qu'au prix de certaines simplifications et ne peut donc pas 
donner un résultat précis. On peut cependant penser qu'il respecte les ordres de grandeur et que son résultat fournit un guide utile pour l'évaluation du comportement d'une couche de verre.

4. Discussion des résultats obtenus. - 4.1 CONDITIONS A SATISFAIRE POUR QUE LA COUCHE DE VERRE SOIT UTILISABLE. - Une constante de temps caractérise chaque phénomène :

- pour l'écoulement sous l'effet d'une accélération

$$
t_{e}=\frac{3 \mu}{\gamma \rho e}
$$

- pour la guérison des fissures

$$
t_{f}=\frac{f \mu}{\sigma}
$$

Pour que la couche de verre remplisse efficacement son rôle dans un revêtement de protection contre l'oxydation, il faut d'une part qu'elle ne s'écoule pas trop vite à la température maximale d'utilisation $T_{\mathrm{M}}$, où le verre est relativement fluide et où elle doit séjourner pendant une durée totale $t_{\mathrm{M}}$; d'autre part, il faut qu'elle puisse guérir ses fissures à la température minimale $T_{\mathrm{m}}$ où cela est nécessaire, qui peut être soit la température minimale d'utilisation, soit la température minimale à laquelle l'oxydation est encore appréciable. Dans chaque cas, la cinétique de l'oxydation impose une limite supérieure $t_{\mathrm{m}}$ à la durée acceptable pour la guérison d'une fissure. Le revêtement sera donc utilisable si les deux conditions suivantes sont satisfaites :

$$
\begin{aligned}
& t_{e} \geqslant t_{\mathrm{M}} \quad \text { soit } \quad \frac{3 \mu\left(T_{\mathrm{M}}\right)}{\gamma \rho e} \geqslant t_{\mathrm{M}} \\
& t_{f} \leqslant t_{\mathrm{m}} \quad \text { soit } \quad \frac{f \mu\left(T_{\mathrm{m}}\right)}{\sigma} \leqslant t_{\mathrm{m}} .
\end{aligned}
$$

4.2 APPlication AU CAS D'UN REVÊTEMENT SITUÉ SUR UNE AUBE DE TURBINE. - A titre d'exemple, on va considérer le cas d'un revêtement situé sur une aube de turbine à gaz travaillant à $1200^{\circ} \mathrm{C}$. On suppose que la turbine a un rayon $R_{t}=40 \mathrm{~cm}$, et tourne à environ 10000 tours/minute $(\omega=1000 \mathrm{rd} / \mathrm{s})$. Une couche de $5 \mu$ d'épaisseur d'un verre de masse spécifique $2 \mathrm{~g} / \mathrm{cm}^{3}$ recouvre la surface de l'aube. La viscosité minimum à donner à ce verre pour que la couche soit stable pendant une durée $t_{\mathrm{M}}=100 \mathrm{~h}$ est :

$$
\mu\left(T_{\mathrm{M}}\right)=\frac{t_{\mathrm{M}} \gamma \rho e}{3}=\frac{t_{\mathrm{M}} \omega^{2} R_{\mathrm{t}} \rho e}{3}
$$

d'où

$$
\mu\left(T_{\mathrm{M}}\right)=4,8 \times 10^{9} \text { poises } .
$$

Cela correspond à un verre très visqueux.
On peut considérer que l'oxydation du niobium ou de ses alliages est déjà appréciable à $500^{\circ} \mathrm{C}$. Si on considère que le rapport des viscosités du verre à $500{ }^{\circ} \mathrm{C}$ et $1200^{\circ} \mathrm{C}$ est de l'ordre de $10^{6}$, on obtient $\mu\left(T_{\mathrm{m}}\right)=4,8 \times 10^{9} \times 10^{6}=4,8 \times 10^{15}$ poises. Si la tension superficielle du verre est de 100 dynes $/ \mathrm{cm}$, la constante de temps de guérison à $500^{\circ} \mathrm{C}$ d'une fissure de $1 \mu$ de large est alors

$$
t_{f}=\frac{f \mu\left(T_{\mathrm{m}}\right)}{\sigma}=\frac{10^{-4} \times 4,8 \times 10^{15}}{100}=4,8 \times 10^{9} \mathrm{~s} .
$$

La stabilité de la couche à $1200^{\circ} \mathrm{C}$ et la guérison des fissures à $500^{\circ} \mathrm{C}$ sont donc incompatibles.

4.3 APPLICATION AU CAS D'UN REVÊTEMENT SITUÉ SUR UNE PIÈCE IMMOBILE TRAVAILlANT A TEMPÉRATURE CONSTANTE. - On suppose que la pièce n'est soumise qu'à l'accélération de la pesanteur : $v=10^{3} \mathrm{~cm} / \mathrm{s}^{2}$.

La viscosité minimale pour qu'une couche de $5 \mu$ d'épaisseur d'un verre de masse spécifique $2 \mathrm{~g} / \mathrm{cm}^{3}$ soit stable pendant une durée de $100 \mathrm{~h}$ est :

$$
\mu\left(T_{\mathrm{M}}\right)=1,2 \times 10^{5} \text { poises } .
$$

Il s'agit là d'un verre relativement fluide.

La constante de temps de guérison à la température $T_{M}$ d'une fissure de $1 \mu$ de largeur est alors (en prenant $\sigma=100$ dynes $/ \mathrm{cm}$ )

$$
t_{f}=0,12 \mathrm{~s} .
$$

Un tel revêtement conviendra donc bien à une pièce dont le programme de travail comprend des séjours à une température élevée et constante, alternés avec des séjours à basse température où l'oxydation n'est pas appréciable, avec des montées ou descentes en température très rapides : la guérison des fissures dues aux chocs thermiques ne sera pas nécessaire à basse température et sera très rapide à haute température.

5. Conclusion et résumé. - Deux phénomènes d'écoulement visqueux se produisent dans une couche de verre d'épaisseur constante déposée sur un substrat solide que le verre mouille parfaitement. Le déplacement du verre sous l'effet des accélérations parallèles à la couche et la guérison des fissures sous l'effet des forces de capillarité. De l'étude de ces deux écoulements et de la comparaison des constantes de temps qui les caractérisent, on peut déduire dans quels cas d'application la couche pourra satisfaire aux deux exigences de stabilité mécanique et d'auto-guérison des fissures: Dans le cas d'une couche déposée sur une aube de turbine à gaz, ces deux exigences ne peuvent pas être satisfaites simultanément; par contre, elles peuvent l'être dans le cas d'une couche située sur une pièce statique travaillant à température constante. 


\section{Notations utilisées :}

$\rho\left(\mathrm{g} / \mathrm{cm}^{3}\right)$

$\mu$ (poise)

$\sigma$ (dyne $/ \mathrm{cm})$

$\gamma\left(\mathrm{cm} / \mathrm{s}^{2}\right)$

$R_{t}(\mathrm{~cm})$

$\omega(\mathrm{rd} / \mathrm{s})$

$e(\mathrm{~cm})$

$O x, O y, O z$ masse spécifique du liquide ;

viscosité du liquide ;

tension superficielle du liquide;

accélération ;

rayon de la turbine ;

vitesse de rotation de la turbine;

épaisseur de la couche de verre ;

système d'axes tel que le plan $x O y$ est la surface du substrat solide. $O x$ est parallèle à l'accélération. La fissure est parallèle à $O y$;

$c(z)\left(\right.$ dyne $\left./ \mathrm{cm}^{2}\right)$ contrainte de cisaillement dans le verre à la cote $\mathrm{z}$, parallèlement à l'accélération et dans le plan parallèle à la couche ;

$v(\mathrm{~cm} / \mathrm{s})$

$t_{e}(\mathrm{~s})$ vitesse d'écoulement du verre;

temps après lequel le déplacement moyen de la couche sous l'effet de l'accélération est égal à son épaisseur ; $f(\mathrm{~cm})$

$t_{f}(\mathrm{~s})$

largeur de la fissure;

temps après lequel une fraction importante de la fissure est remplie de verre ;

$C_{\mathrm{AB}}\left(\right.$ dyne $\left./ \mathrm{cm}^{2}\right)$ contrainte de cisaillement parallèle à $\mathrm{AB}$, sur le plan $\mathrm{ABy}$;

$P_{\mathrm{AB}}\left(\right.$ dyne $\left./ \mathrm{cm}^{2}\right)$ contrainte perpendiculaire au plan $\mathrm{AB} y$,

$P_{\mathrm{AB}}$ est positif s'il s'agit d'une compression ;

$V\left(\mathrm{~cm}^{3}\right) \quad$ volume ;

$T_{\mathrm{M}}\left({ }^{\circ} \mathrm{C}\right) \quad$ température maximale d'utilisation du revêtement ;

température minimale à laquelle le revêtement doit protéger le substrat contre l'oxydation;

$t_{\mathrm{M}}(\mathrm{s}) \quad$ durée du séjour à $T_{\mathrm{M}}$;

$t_{\mathrm{m}}(\mathrm{s}) \quad$ durée maximale du séjour à $T_{\mathrm{m}}$ au bout duquel l'oxydation du substrat par une fissure ouverte est encore tolérable.

\section{Bibliographie}

[1] Stecher (P.), Lux (B.), Funk (R.), Protection contre l'oxydation d'alliages de niobium, Entropie, 1968, 39.

[2] Lux (B.), Stecher (P.), Perlhefter (N.), Protection contre l'oxydation du niobium au moyen d'une couche de $\mathrm{NbSi}_{2}$ imprégnée de $\mathrm{Sn}-\mathrm{Al}$, Annales des Mines, 1966, 802.

[3] Stecher (P.), Lux (B.), Protection du niobium contre l'oxydation, Publication à paraître simultanément avec le présent texte, $J$. Physique, 1970, 5, 573. 